\title{
Aromatherapy in Textiles: A Systematic Review of Studies Examining Textiles as a Potential Carrier for the Therapeutic Effects of Essential Oils ${ }^{\dagger}$
}

\author{
Sunidhi Mehta ${ }^{1, *}$ and Maureen MacGillivray ${ }^{2, \ddagger(D)}$ \\ 1 Davis School of Design and Community Development, West Virginia University, Morgantown, WV 26505, USA \\ 2 Department of Fashion, Interior Design and Merchandising, Central Michigan University, \\ Mount Pleasant, MI 48858, USA; macgi1ms@gmail.com \\ * Correspondence: sm00038@mail.wvu.edu \\ + Scientific Article No: 3421 of the West Virginia Agricultural and Forestry Experiment Station, Morgantown. \\ $\ddagger$ Retired, current address: 1216 Clubhouse Drive, Lake Isabella, MI 48893, USA.
}

check for

updates

Citation: Mehta, S.; MacGillivray, M.

Aromatherapy in Textiles: A

Systematic Review of Studies

Examining Textiles as a Potential

Carrier for the Therapeutic Effects of

Essential Oils ${ }^{\dagger}$. Textiles 2022, 2, 29-49.

https://doi.org/10.3390/

textiles2010003

Academic Editors: Selestina Gorgieva and Andrea Zille

Received: 22 November 2021

Accepted: 1 January 2022

Published: 6 January 2022

Publisher's Note: MDPI stays neutral with regard to jurisdictional claims in published maps and institutional affiliations.

Copyright: () 2022 by the authors. Licensee MDPI, Basel, Switzerland. This article is an open access article distributed under the terms and conditions of the Creative Commons Attribution (CC BY) license (https:/ / creativecommons.org/licenses/by/ $4.0 /)$.

\begin{abstract}
Integrative medicine is a rapidly growing specialty field of medical care that emphasizes the amalgamation of complementary therapies and conventional medicine. Aromatherapy, one of the complementary therapies, is a centuries-old tradition, used in many cultures and societies as an alternative to, or in conjunction with, conventional medicine. However, there is very little understanding of its therapeutic benefits in the scientific realm related to the correct dosage of essential oils, their delivery mechanism and their efficacy on human physiology in general. We reviewed studies published between 2011-2021 focused on aromatherapy and textiles, and explore "textile" materials as a possible carrier for essential oils in this paper. Due to their proximity to the biggest organ of the human body, textiles can potentially serve as a good delivery system for the therapeutic benefit of essential oils. After this rigorous review, we found gaps in the field. Therefore, we propose cross-disciplinary synergies for future research to fully understand the therapeutic efficacy of essential oils.
\end{abstract}

Keywords: aromatherapy; textiles; aromachology; microencapsulation; aromatherapeutic textiles; bio-functional textiles; integrative medicine; essential oils

\section{Introduction}

Aromatic plants are a class of plants that contain fragrant compounds or essential oils (EO). The EOs can be sourced from petals and flowers, grasses, seeds, stems, leaves, needles, rinds and fruits, roots and rhizomes, woods, and resins. They are overly complex, volatile liquids including terpenes, sesquiterpenes, oxygenated derivatives, aldehydes, oxides, phenols, ethers, acids, and ketones [1]. The earliest reference to essential oils such as sandalwood and cinnamon for human health and wellness dates to around $1200 \mathrm{BC}$, found in the ancient Hindu scriptures called the Vedas. Likewise, a written order for "imported oil of cedar, myrrh and cypress" was found on a clay tablet believed to be from Babylon dating to $1800 \mathrm{BC}$ [2]. Over 3500 years ago, the Egyptians were using plants for medicine, healing massage, surgery, food preservation and mummification [3]. Such practices were also used by the Greeks and Romans who added their own rituals of fragranced baths and daily massages with fragranced oils [4].

Today, the practice of using essential oils for its positive effect on mood, behavior and wellness is known as "aromatherapy". The term aromatherapy was coined in 1928 by a French chemist, René-Maurice Gattefossé, who was interested in the use of essential oils for medicinal purposes. His work [5], and the use of aromatic essences to treat patients in civilian and military hospitals, reintroduced essential oils into modern medicine. Today, aromatherapy is defined as the skilled and controlled use of essential oils for physical and 
emotional health and wellbeing [6]. To bring a more scientific approach to the examination of the effects of essential oils, the term "aromachology" was coined in 1982 by the Sense of Smell Institute. Aromachology is defined as the scientific analysis of olfactory effects on mood, physiology, and behavior. Although the term 'aromachology' did not replace the term 'aromatherapy' in the scientific or commercial realms, the new nomenclature did appear to bring more attention to the importance of performing empirical studies on the biological effects and health benefits of essential oils.

\subsection{Revival of Aromatherapy}

The use of essential oils for health and wellness has seen a resurgence in the late 20th and early 21st centuries. Many consumers are showing a renewed interest in following a healthy lifestyle that includes natural products and the use of alternative medicines. In fact, acceptance of alternative methods to treat medical conditions, such as pain, anxiety, depression, and insomnia with plant-based essential oils is increasing in the world of western medicine [7]. The use has gained popularity due to its cost-effectiveness, fewer-to-no side effects compared to drug therapies, and improvement of patients' overall sense of health and wellbeing [8]. While the therapeutic use of aromatic essential oils is a centuries-old tradition in many cultures, it remains an important part of the holistic approach of eastern medicine. It is one of the fastest-growing complementary therapies worldwide. According to the National Institutes of Health-National Center for Complementary and Integrative Health (NCCIH), Americans spend more than $\$ 30.2$ billion annually on aromatherapy [9]. It is predicted that the global market for aromatherapy will grow in spending to $\$ 5$ trillion by 2050 [10].

In the second decade of the 21st century, aromatherapy has shown significant medical effects in studies on dementia [11]; anxiety and depression [12]; pain management [13]; dysmenorrhea [14]; and sleep disorders [8]. While these specific studies show promise, many research studies that claim to demonstrate the effect of aromatic compounds on human physiology and psychology are vexed with problems. Scientific evidence of the therapeutic benefits of essential oils on human health remains sparse.

\subsection{Effect of Aromatic Compounds on Our Senses}

Aromatic compounds work on our physiology and senses in many ways. Examples of consumer products that contain aromatics are perfume/cologne, essential oil plug-ins, candles, scented aerosol sprays, fabric softeners, beauty products and food flavorings. Their route into the body can be through inhalation via the olfactory system or by oral ingestion. Upon inhalation of the essential oil aroma, its molecules activate the olfactory, gastrointestinal and/or integumentary systems based on the pathway of activation. These molecules are capable of releasing neurotransmitters such as endorphins to trigger a sense of wellbeing and an analgesic effect $[7,8]$. Several plant-based essential oils have therapeutic effects on our body and senses; in fact, the discovery of acetyl salicylic acid, the active ingredient in aspirin, is linked to the chewing of willow bark to alleviate pain, inflammation, and fever [8]. Numerous studies have evaluated the effects of aromatherapy on human physiology [15-18]. Nonetheless, aromatherapy was never accepted as part of mainstream medicine due to the lack of supporting evidence for its effectiveness. This paper is aimed at studying the current state of aromatherapeutic textiles using relevant literature and investigating how the current gaps in the field can be filled through future research on this topic.

\section{Selection of Literature}

The literature on "aromatherapy and textiles," "therapeutic and textiles," fragrance and textiles," "scent and textiles," and "essential oil and textiles" was searched using the PubMed and Web of Science databases. The PubMed database was used to capture biomedical and life sciences journal literature on these topics at the U.S. National Institutes of Health and National Library of Medicine. The Web of Science database was mined to 
include scholarly research journal articles found in the sciences, social sciences, arts, and humanities. Our initial search resulted in 303 studies (see Figure 1) in the field of aromatherapy and its application in textiles that were published between the years 2011-2021. We used the following criteria to further scrutinize our search results and select relevant literature for review. Studies that met at least $3 / 5$ of the following criteria were included into the "acceptable/qualified" category:

1. The study must have well-directed goals/objectives.

2. Therapeutic effects of the fragrances were tested using a suitable experimental methodology.

3. The study had a sample representative of sufficient subject population OR the sample studies were selected from credible database sources for review papers.

4. Data were analyzed using appropriate statistical methods.

5. The study was published in a peer-reviewed journal.

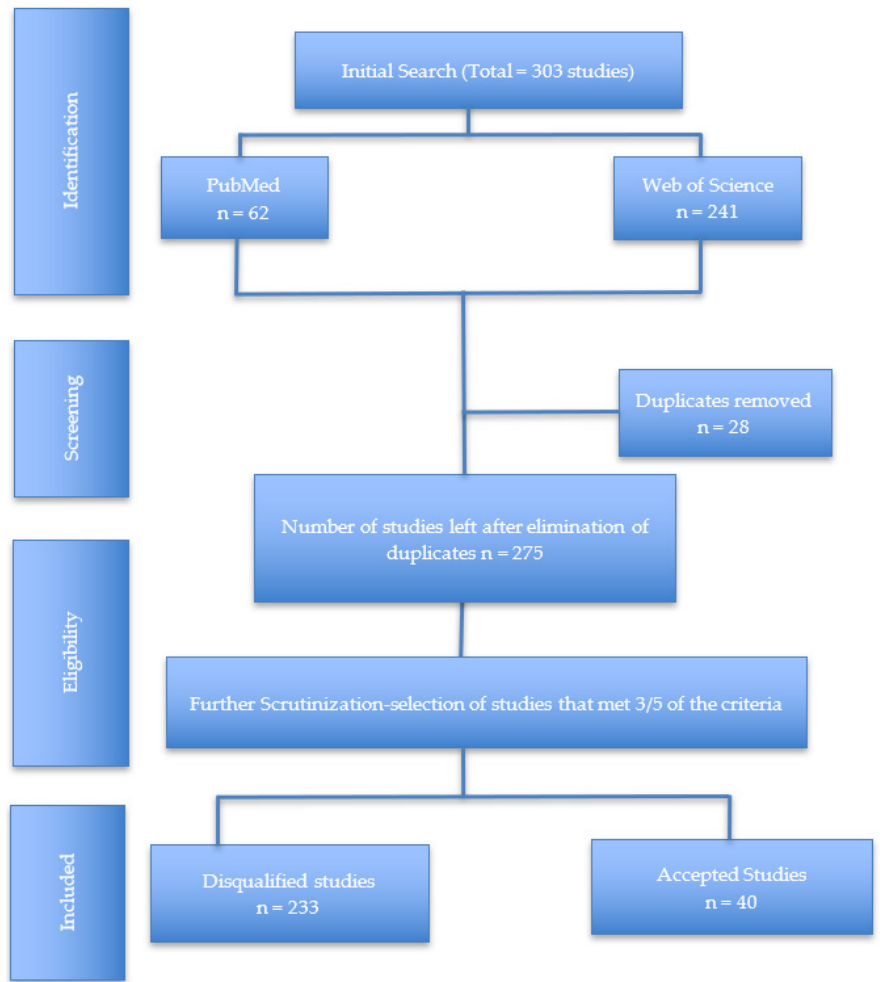

Figure 1. Selection of literature for review.

Studies that did not meet the selection criteria went into the "discarded/disqualified" category. In the end, we had 40 acceptable studies for review. We summarized the selected studies in the Table 1 below.

The Table 1 demonstrates the wide variety of research being conducted in this field. The publications include the fields of chemistry, biology, agriculture, medicine, engineering, fashion, textiles, public health, coloration and materials. While the foundation for research on aromatherapy and textiles is broad, the table exhibits opportunities for crossdisciplinary research that not only will advance individual fields of study, but the potential for improving human health and wellness. 
Table 1. Summaries of the aromatherapy studies reviewed for this paper.

Authors

1. Ghayempour \& Montazer [1]

2016

J. Microencapsul.

Micro/Nanoencapsulation of
Dsential Oils and Fragrances: Focu

on Perfumed, Antimicrobial
on

Mosquito-Repellent and Medical Textiles.

Key Findings

The article reviews the micro/nanoencapsulated essential oils with a focus on perfumed, anti-microbial, mosquito-repellent and medical textiles. The

biggest challenge in the field of aromatherapy is the determination of the

right dosage and high volatility of the aromatic compounds that can be overcome by encapsulating the essential oils. Multifunctional

aromatherapeutic textiles hold great potential for future research.

Clinical aromatherapy can be beneficial for pain, nausea, vomiting, anxiety, sadness, stress, sleeplessness, agitation with dementia, cancer pain, and

end-of-life symptoms. It has been demonstrated to be effective in a variety of contexts, including critical care, oncology, palliative care, hospice, and

2. Farrar \& Farrar [7] $2020 \quad$ Nurs. Clin. N. Am. Clinical Aromatherapy surgery. Aromatic essential oils, on the other hand, can be hazardous and toxic since some oils are flammable, cause skin dermatitis, or are phototoxic, posing a risk of chemical burn, oral poisoning, and even death. Therefore, the right dosage and delivery method must be considered while administering aromatherapy.

Aromatherapy interventions for improved quality of sleep were explored and found to be helpful in enhancing sleep quality in a meta-analysis of 12

studies using a random-effects model. In both ill and healthy patients, $2015 \quad$ J. Altern. Complement. Med. $\quad \begin{gathered}\text { The Effects of Aromatherapy } \\ \text { on Sleep }\end{gathered}$ than massage therapy. As a result, special recommendations for the effective use of aromatherapy must be developed.

The antimicrobial activity of essential oils derived from different medicinal and aromatic plants against diverse bacterial, fungal, and viral infections was studied. Essential oils have great potential in the field of biomedicine due to their biocidal properties, which allow them to disrupt pathogen cell

Evid. Based Complement. Altern. Med.
Antimicrobial Properties of Plan Essential Oils against Human Pathogens and Their Mode of Action: An Updated Review membranes. Exploration of the molecular mechanisms of essential oils and their specific chemical constituents should be the focus of future research.

The effect of aromatherapy on agitation and behavioral symptoms was found

to be inconsistent. When aromatherapy was compared to a placebo, no difference in deleterious effects was found. Further well-designed,

5. Forrester et al. [11]

2016 Cochrane Database Syst. Rev. Aromatherapy for Dementia.

arge-scale randomized controlled trials are required to form any firm conclusions about the efficacy of the aromatherapy in treating dementia. 
Table 1. Cont.

\section{Authors}

Date

Journal Title

Paper Title

\section{Key Findings}

A quantitative-structure activity relationship (QSARs), was developed to predict skin-and-blood-barrier penetrative abilities. The researchers studied 119 terpenoids from EOs used in aromatherapy for the QSAR model
Models for Skin and Brain

6. Agatonovic-Kustrin et al. [12] $2020 \quad$ J. Biomol. Struct. Dyn.

from Essential Oils Used in Penetration of Major Components Aromatherapy for Dementia Patients

The Effectiveness of Aromatherapy in Reducing Pain: A Systematic Review and Meta-Analysis

Effects of Aromatherapy on

Dysmenorrhea: A Systematic Review and Meta-Analysis development. The QSAR model for blood brain barrier permeability was dominated by well-known characteristics such as $\log$ P (lipophilicity), molecule size, and shape. Hydrocarbon terpenes with the lowest molecular size and maximum lipophilicity were found to have the highest anticipated barrier penetration ability. As a result, molecule size is shown to be a barrier to penetration. Furthermore, skin permeability is increased by compounds with a slightly larger molecular size, high lipophilicity, and low polarity.

A meta-analysis of 12 studies on aromatherapy related to pain management was conducted in this paper. Aromatherapy was found to have a considerabe positive effect in relieving pain in this study. The findings of the study suggest that aromatherapy should be regarded as a safe supplement to current pain management treatments.

The efficacy of aromatherapy in relieving pain associated with dysmenorrhea was studied. The findings suggest statistically significant relief from pain in the treatment group that received aromatherapy for pain relief compared to the control group that was given a placebo treatment.

This interesting article discusses the discovery of aspirin-an important pharmaceutical drug used in cardiovascular disease prevention and pain relief. The discovery of the drug was linked to the use of willow bark as traditional medicine for more than 3500 years.

A systematic review of aromatherapy literature was conducted by searching

Aromatherapy for Health Care: An Overview of Systematic Reviews
12 electronic databases without restrictions on time or language. A majority
of the studies were related to measuring the efficacy of aromatherapy for

hypertension, depression, anxiety, pain relief, and dementia. Due to a number of caveats, the evidence is not sufficient to conclude that aromatherapy is an effective therapy for any condition. 
Table 1. Cont.

Authors

Journal Title Paper Title

\section{Key Findings}

The Effects of Aromatherapy

11. Won \& Chae [16]

2011

J. Korean Biol. Nurs. Sci.
Massage on Pain, Sleep, and Stride

Length in the Elderly with Knee

Osteoarthritis

After the aromatherapy massage, there was a significant difference in pain scores between the experimental and control groups. There were no significant variations between the two groups' sleep scores. The stride length of the experimental group was substantially longer than that of the control group. In the elderly with knee osteoarthritis, aromatherapy massage could be advised as an effective technique to reduce discomfort and increase stride length.

The aromatherapy group had a reduced median pain score in the latent and early active phases. In the aromatherapy group, the mean changes in pain scores between the latent and early active phases, as well as the baseline, were considerably lower. Pain levels in the late active phase and other

Efficacy of Aromatherapy for Reducing Pain during Labor: A Randomized Controlled Trial

Effects of Aromatherapy Combined with Music Therapy on Anxiety, Stress, and Fundamental Nursing Skills in Nursing Students: A

Randomized Controlled Trial lowering pain in the latent and early active phases, and it is likely that it can be utilized as an auxiliary approach for labor pain relief without major adverse effects.

Public Health

13. Son et al. [18]

2019

When compared to aromatherapy and music therapy as individual therapies, the combination had a substantial effect on test anxiety, state anxiety, stress, and the performance of fundamental nursing skills. The findings imply that aromatherapy paired with music therapy in nursing education may be usefu in strengthening essential nursing skills and reducing anxiety and stress among nursing students.

When depositing polymeric compounds on textile surfaces, the comfort indicators (air and water vapor permeability) showed lower values than the untreated fabric, which was as predicted. The pre-cat ionized samples

Essential Oil Microcapsules Immobilized on Textiles and Certain Induced Effects showed a larger reduction in this feature. All evaluated samples, however,

met the minimum standards for skin contact clothing items and home

apparel in terms of air and water vapor permeability. The rose/sage

microcapsule functionalization treatments sustained five washing cycles with acceptable washing durability. 
Table 1. Cont.

\begin{tabular}{|c|c|c|c|c|}
\hline Authors & Date & Journal Title & Paper Title & Key Findings \\
\hline 15. West \& Annet-Hitchcock [21] & 2014 & $\begin{array}{l}\text { J. Text. Appar. } \\
\text { Technol. Manag. }\end{array}$ & $\begin{array}{c}\text { A Critical Review of Aroma } \\
\text { Therapeutic Applications } \\
\text { for Textiles }\end{array}$ & $\begin{array}{l}\text { A review of aromatherapeutic textiles research was conducted. Due to } \\
\text { interest in sustainability, environmental consciousness and healthy living, } \\
\text { aromatherapy is gaining popularity. There is a strong potential for } \\
\text { aromatherapeutic textiles with multifunctionality such as comfort, } \\
\text { antimicrobial activity, and relaxation benefits. }\end{array}$ \\
\hline 16. Mehta \& MacGillivray [22] & 2016 & AATCC J. Res. & $\begin{array}{l}\text { Analysis of Stress Relief Effect by } \\
\text { Use of Aroma-Therapeutic Textiles }\end{array}$ & $\begin{array}{l}\text { Aromatherapeutic textiles finished with lavender and cedarwood oil-based } \\
\beta \text {-cyclodextrin inclusion complexes were developed for stress relief. The } \\
\text { stress-relief efficacy of the aromatherapeutic textiles was measured by the } \\
\text { autonomous nervous system response of human subjects to the } \\
\text { aromatherapeutic textiles. There was a statistically significant difference in } \\
\text { stress relief among the treatment group compared to the control group. } \\
\text { However, there was no significant difference between the stress relief effects } \\
\text { of lavender and cedarwood oil. }\end{array}$ \\
\hline 17. Golja et al. [23] & 2013 & Color. Technol. & $\begin{array}{l}\text { Fragrant finishing of cotton with } \\
\text { microcapsules: comparison between } \\
\text { printing and impregnation }\end{array}$ & $\begin{array}{l}\text { Both application processes are adequate for effective aromatic but ineffective } \\
\text { antibacterial finishing of cotton garments, according to the findings. The } \\
\text { microcapsules were unaffected by artificial light. Only when the capsules are } \\
\text { printed on cloth can they influence the color. Both printing and impregnation } \\
\text { procedures have advantages and disadvantages. }\end{array}$ \\
\hline 18. Golja \& Tavcer [24] & 2016 & Tekstilec & $\begin{array}{c}\text { Textile Functionalisation by Printing } \\
\text { Fragrant, Antimicrobial and } \\
\text { Flame-Retardant Microcapsules. }\end{array}$ & $\begin{array}{l}\text { The printing technology can be used to generate a variety of fabric properties. } \\
\text { It was found that } 100 \mathrm{~g} \text { of suspension ( } 32 \mathrm{~g} \text { of microcapsules) per kilogram of } \\
\text { fabric was the best concentration of microcapsules for producing the required } \\
\text { aromatic or antibacterial textile product. The necessary fire-retardant } \\
\text { material required a very high concentration of microcapsules, which could } \\
\text { not be accomplished using the pigment technique. }\end{array}$ \\
\hline 19. Specos et al. [25] & 2011 & J. Ind. Text. & $\begin{array}{c}\text { Aroma Finishing of Cotton Fabrics } \\
\text { by Means of Microencapsulation } \\
\text { Techniques }\end{array}$ & $\begin{array}{l}\text { The authors used microencapsulation technique to develop functional } \\
\text { textiles with added value in cotton fabric. Microcapsules were obtained by } \\
\text { complex coacervation using gelatin and arabic gum or by encapsulation in } \\
\text { yeast cells in order to increase the durability of fragrances in textiles. } \\
\text { Microcapsule characterization, such as particle size and morphology, was } \\
\text { carried out for different oils to polymer ratios and hardening agents to } \\
\text { polymer ratios. The fragrance release was tested using an electronic nose. }\end{array}$ \\
\hline
\end{tabular}


Table 1. Cont.

Authors Date

Date

Journal Title

Paper Title

Controlled release of the essential

oil of citronella microencapsulated

using cotton and polyester matrices

20. Bezerra et al. [26] $2016 \quad \begin{array}{ll}\text { Cellulose } & \begin{array}{c}\text { oil of citronella microencapsulated } \\ \text { using cotton and polyester matrices }\end{array}\end{array}$

21. Manfredini et al. [27]

2020

Ind. Eng. Chem. Res.

Polymer Nanoparticles for the Release of Fragrances: How the

Physicochemical Properties

Influence the Adsorption on Textile and the Delivery of Limonene

Preparation and Characteristics of

22. Liu et al. [28]

2014

Flavour Fragr. J.

Nanocapsules Containing Essential

Oil for Textile Application

\section{Key Findings}

The kind of fiber had a direct impact on the release; microcapsules in polyester showed Fickian diffusion, whereas modified cotton had a non-Fickian kinetic model. Understanding regulated release processes is critical for establishing and developing more long-lasting finishing effects.

The surface charge was found to be the most critical feature in driving adsorption, with positively charged NPs having a larger Qe than their negatively charged counterparts, owing to the cotton fibers' overall negative surface. The Langmuir type isotherm of these NPs emphasizes the favorable nature of the process. Furthermore, the degree of cross-linking does not appear to have impacted the number of NPs adsorbed on the textile considerably, at least at the concentration utilized in this work. $\mathrm{Tg}$, on the other hand, plays an important effect, with soft PBA NPs adsorbing more than tougher PBMA and PSTY NPs. Finally, larger NPs outperformed smaller ones in terms of adsorption, but not in terms of limonene encapsulation efficiency.

Methyl Methacrylate Styrene copolymer nanocapsules containing cologne essential oil for fabric treatment were prepared by miniemulsion

polymerization. FT-IR spectroscopy, TGA, and SEM images were used to examine the morphology, chemical composition and thermal properties of the fabric. Encapsulation efficiency was $85 \%$ with loading capacity at $42 \%$. After 15 washings, $6.8 \%$ of the cologne remained, indicating good washing durability.

Microwave Curing for Applying Polymeric Nanocapsles Containing

23. Ghayempour and Mortazavi [29]
Cellulose
Essential Oils on Cotton Fabric to

Produce Antimicrobial and Fragrant Textiles
Alginate nanocapsules containing peppermint oil prepared by microemulsion were applied to cotton fabric to examine washing durability and antimicrobial activity. FT-IR spectroscopy, TGA, and SEM images were used to examine the surface distribution and position of the nanocapsules on the fabric. Sixteen percent of the peppermint oil remained after 25 washings. In terms of antimicrobial activity, a 100\% reduction in bacteria was demonstrated for both E. coli and S. aureus bacteria. 
Table 1. Cont.

\section{Authors}

Date

Journal Title

Paper Title

Scent Properties by Natural

24. Sánchez-Navarro et al. [30]

2015

Polym. Int.

Fragrance Microencapsulation for

Footwear Applications

\section{Key Findings}

The delivery behaviors of the produced microcapsules were characterized in two ways. On the one hand, because melamine-formaldehyde microcapsules are more robust, they can be used in materials such as linings that are subjected to strong mechanical and thermal loads. Gelatin-carboxymethyl cellulose microcapsules, on the other hand, should be used in lower-stress footwear components, such as insoles, because they are less robust and may not withstand the process conditions. The use of both types of microcapsules could result in fragrance release that is both quick and long-lasting.

Astaxanthin's biological applicability is limited due to its unstable structure.

This problem can be solved by converting astaxanthin to astaxanthin esters or encapsulating it. In this article, the advantages and disadvantages of various encapsulating agents andprocedures were explored. As a result, more research should be carried out to see how encapsulated astaxanthin affects various biological functions and how it might be used in the nutraceutical and pharmaceutical industries.
25. Suganya \& Anuradha [31] $2017 \quad$ Int. J. Pharm. Clin. Res. $\quad \begin{gathered}\text { Microencapsulation and } \\ \text { Nanoencapsulation: A Review }\end{gathered}$
IOSR J. Polym. Text. Eng.
Microencapsulation for Textile Finishing

Micro-encapsulation is a process in which small capsules with many useful properties are made by using tiny particles or droplets surrounded by a coating. The material inside the microcapsule is called the core material whereas the wall is called a shell, coating, or membrane. Usually, microcapsules have diameters between a few micrometers and a few millimeters. Many special and functional properties can be imparted to the fabrics by microencapsulating the core material. Encapsulation has allowed moisturizers, therapeutic oils, and insecticides to be incorporated into fabrics.

Molecular Encapsulation of Lavender Essential Oil by 27. Khajavi et al. [33] 2013

\section{Lavender bonded to B-cyclodextrin, consistent with their mutual}

hydrophobic character, and treated samples with the encapsulated fragrance compound showed greater durability in comparison with untreated and DMDHEU-treated samples. Water sorption in cellulosic fabrics is reduced when DMDHEU is used. Body sweat (fatty acid) replaces lavender and causes it to be released; body sweat fills the cavity of b-cyclodextrins.
Fragrance Finishing of Cotton Fabrics 
Table 1. Cont.

Authors
Journal Title

Paper Title

\section{Key Findings}

Aromatherapy essential oils were sprayed inside Cyclodextrin's oleophilic cavities. The smells lasted at least six washes and six weeks after application; nevertheless, they continued to give off an aroma long after the last

Embedding Aromatherapy Essential Oils into Textile Fabric Using $\beta$-Cyclodextrin Inclusion Compound

28. Ghosh [34]

2015

Indian J. Fibre Text. Res.

assessment. The perfume intensity of the polyester/cotton fabric was found

to be higher, which was assumed to be due to a higher loading of the Cyclodextrin, which contained more aromatherapy oil in the cavities. Boost the amount of Cyclodextrin in the pores of the sol-gel to increase the scent's intensity and longevity.

Due to weak forces of interaction between the oils and cotton, the application of essential oils without any form of anchoring hosts lasted only a few hours The use of just Cyclodextrin resulted in a slower but longer release of oils.

The use of MCT-CD showed exceptional results, with exceptionally

Performance assessment of

Fash. Text. fragrance finished cotton with cyclodextrin assisted anchoring hosts persistent essential oil retention enduring five or more washes and even for a longer period. As a result, MCT-CD was proven to be a more useful host for long-lasting functionalized materials. Tensile strength had increased with MCT-CD (pH 6 and 8); on the other hand, air permeability had decreased, stiffness had increased, and oil stability had increased with the changed host.

The data on the general characteristics and complexing ability of cyclodextrins, as well as assessment methodologies, are presented in this work. It focuses on the creation of drug deposits on the surface of a textile underlayer utilizing a cyclodextrin chemical that favors the incorporation of a medication/active principle and their release onto the dermis of patients with skin problems or for insect protection. It also includes the cyclodextrin drug's kinetics, duration, diffusion flow, and release media for in vitro research, as well as the active principle's release modeling. Antibacterial, anti-allergic, antifungal, chronic venous insufficiency, psoriasis, and insect protection are among the topics covered. The active

ingredients/pharmacodynamic agents used on cotton, woolen, and synthetic textiles are discussed. 
Table 1. Cont.

Authors Date

Journal Title

A Review of e-Textiles in
Neurological Rehabilitation: How

31. Mclaren [37]

J. Neuroeng. Rehabil. Close Are We?

\section{Key Findings}

Motion sensing and the measurement or activation of muscle activity are two areas of research highlighted in the review. E-textiles appear to be able to consistently measure gross movement and whether an individual has attained a predetermined movement pattern when it comes to motion sensing. However, the technology is currently laborious and lackluster in terms of resolution. The evaluation found a scarcity of high-quality clinical evidence as well as, in some cases, clinical applicability. Clinical involvement in e-textile research and the use of their experience to produce products that augment and enhance neurological rehabilitation therapy could help to solve these problems.

Mosquito repellents and other pest control products must be tested in the contexts and situations where they will be used, according to the findings of this study. Repellents targeting Aedes, Anopheles, Culex, and other
Patent Literature on Mosquito

32. Pohlit [38]

2011

Planta Med.
Repellent Inventions Which Contain

Plant Essential Oils—a Review mosquito species occurring locally and regionally must be produced using plant oils and separated chemical components. Sprays, fumigants, paints and varnishes, incense, candles, and other household repellents should be used,

as should fumigation and spraying in outdoor settings, and topical repellents, garments made of repellent textiles, repellent wristbands, and other accessible goods should be used for individual protection.

Essential oils, despite their many uses, are extremely sensitive to environmental variables when utilized as such. Encapsulation has emerged as a viable option for improving the stability of essential oils. Various strategies have been employed effectively to achieve this goal, with intriguing outcomes. Other benefits of encapsulating essential oils in particles or liposomes include increased potency and long-term release. Nowadays, the combination of essential oils and active compounds is garnering a lot of interest to produce colloidal particles, which are used mostly in dermatology, local skin therapy, and now in cosmetic textiles as a novel application. 
Table 1. Cont.

Authors Date

Journal Title

Paper Title

Nitrogen and Phosphorus Levels

34. Chrysargyris [40]

2016

Ind. Crops Prod.

Affected Plant Growth, Essential Oil

Composition and Antioxidant

Status of Lavender Plant (Lavandula angustifolia Mill)

\section{Key Findings}

The current findings show that mineral ratios and amounts might have a beneficial or negative impact on aromatic plant development and essential oil production. Both nitrogen and phosphorus are essential nutrients for plant growth and development. The effects of nitrogen and phosphorus rates on hydroponically grown lavender plants were investigated for plant growth, mineral uptake, antioxidant activity, and essential oil constituent alterations under regulated nutrition conditions.

Plant development was mostly impacted by P levels, but lower N levels (150 $\mathrm{mg} / \mathrm{L}$ ) lowered chlorophyll concentration. Lavender's antioxidant status benefited from $\mathrm{N}$ levels greater than $200 \mathrm{mg} / \mathrm{L}$, whereas intermediate $\mathrm{P}$ concentrations encouraged stronger antioxidant action.

Thymol (50.5 percent), borneol (16.7 percent), and carvacrol (7.7\%) were

GC-MS Analysis and in Vitro Antioxidant and Enzyme Inhibitor

Activities of Essential Oil from

Aerial Parts of Endemic Thymus spathulifolius Hausskn used as a thymol source. The essential oil had mild antioxidant and enzyme inhibitory properties in general. This is the first study to show that this oil has antioxidant and enzyme inhibitory properties. T. spathulifolius could be used as a natural source for isolating its active components for use in dietary supplements and pharmaceuticals.

Thermo-sensitive microparticle composition ensured stability during storage while also triggering thermo-sensitive release upon skin contact. The lipid microparticles enabled for medication dispersion and skin diffusion in the

upper layers of the skin, which is ideal for treating superficial fungal

infections and avoiding systemic absorption. Secondly, the customized screen-printing method 535 for the microparticle drug formulation was able

to maintain the drug's pharmacological activity. ECN efficacy was

maintained in vitro on a wide spectrum of fungus strains, while ECN textiles provided high therapeutic efficacy in mice with Cutaneous candidiasis. Overall these findings indicated that this technique has the potential to be used to

manufacture pharmacological textiles for the treatment of superficial infections. These fabrics could be used in bandages or socks to promote patient compliance by making them easier to use. 
Table 1. Cont.

Authors Date Journal Title $\quad$ Paper Title Findings

\begin{tabular}{|c|c|c|c|}
\hline 37. Jaeger [43] & 2015 & Acta Derm.-Venereol. & $\begin{array}{c}\text { Acid-Coated Textiles (PH } \\
\text { 5.5-6.5)—a New Therapeutic } \\
\text { Strategy FOR Atopic Eczema? }\end{array}$ \\
\hline
\end{tabular}

Cotton fabrics with an acid coating were not less comfortable to wear. There were no changes in wearing comfort between the acid-modified and untreated sites of the cotton material. Except for one patient, everyone rated the wearing comfort as excellent or good. There was also no indication of erythema, irritation, or eczema flare-ups.

The oils derived from I. polyneura were found to have significant antibacterial action against Gram-positive bacteria. Prostate cancer cells were more

Interference of Seasonal Variation on the Antimicrobial and Cytotoxic Activities of the Essential Oils from the Leaves of Iryanthera polyneura in the Amazon Rain Forest.

Effects of multiple washing on cotton fabrics containing berberine microcapsules with anti-Staphylococcus aureus activity hazardous than breast cancer cells to the oils. The EOs extracted from leaves gathered during the rainy season were more effective against microbes and were cytotoxic to breast cancer cells. The antibacterial and cytotoxic effects of essential oils extracted from I. polyneura leaves are affected by seasonality, which appears to alter the terpene content of the oils.

The quantity of microcapsules on cotton samples dropped steadily with increasing washing cycles, according to SEM pictures and Fourier transform infrared analyses. Cotton fabrics with agar-gelatin (water-in-oil) microcapsules containing berberine continued to exhibit anti-S. aureus activity after 20 washing cycles. However, only in the contact areas did the chitosan-based (oil-in-water) solution exhibit any growth inhibition against $S$. aureus.

Based on the research available so far, it appears that the essential oil of Litsea cubeba (EOLC) can be used to synthesize substances such as pseudo-ionone, pseudonoe, iodine, methyl iodine, Vitamin E, and Vitamin A. The perfume, scent, culinary flavor, anti-inflammatory, anti-bacterial, and antifungal

Open J. For.
Chemistry of the Main Component of Essential Oil of Litsea cubeba and Its Derivatives 


\section{Mechanism for the Delivery of Aromatic Compounds}

The most important question for the field of aromatherapeutic textiles is, "how can the therapeutic benefits of essential oils be delivered to the human body for long-term symptom relief?" One of the obvious ways to tackle the release of aroma is to encapsulate the essential oils. We reviewed papers in this area to study the latest advancements in the field of EO encapsulation and the environmental considerations.

\subsection{Encapsulation of Aromatic Compounds}

With the essential oils being hydrophobic, formulations have been developed to protect their fragrance, bioavailability and pharmaceutical effects [20]. Encapsulation is the most common carrier mechanism, involving building a shell or wall material that entraps the aromatic compound in a miniature sealed capsule. The capsules can be designed to release their contents under specific chemical, physical, or mechanical conditions making it possible to receive the long-term benefit of the aroma at a controlled rate of release (See Figure 2). Encapsulation of essential oils can be developed at micro or nano levels. The technology of nanoencapsulation is growing exponentially and is used in a variety of industrial applications in addition to textiles, such as the food industry, cell immobilization, fermentation, drug delivery, cell transplantation and many more [18,20]. Food and pharmaceutical industries are currently using nanoencapsulation techniques to increase the storage time of volatile active ingredients. The microencapsulation technique also provides targeted release opportunities for the active ingredients in pharmacological drugs. Similarly, textiles embedded with microencapsulated essential oils can be effective in delivering the therapeutic benefits of these essential oils [21,22].

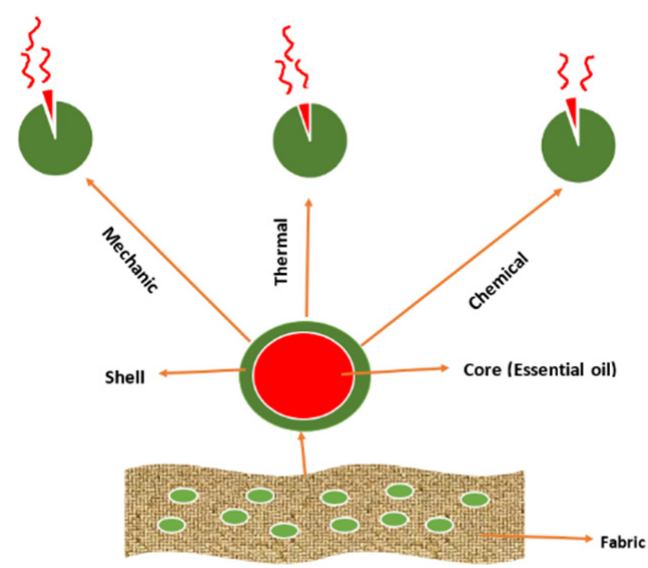

Figure 2. Slow-release mechanism of encapsulated essential oils in aromatherapeutic textiles.

Currently, microencapsulated aroma-textiles are developed mainly for their cosmetological or antimicrobial functions and their therapeutic benefit is rarely measured in empirical studies. We found this to be a significant gap in the literature search performed for the purpose of this paper. Interdisciplinary synergies between researchers from different fields such as health sciences, textile science, consumer behavior, agricultural sciences and nanoscience are required to decipher the best approach to impregnate EO in textiles and to yield their long-term therapeutic benefits. Thus far, many researchers from these fields have worked in silos to advance the research in their own field. However, the literature on developing textiles with aromatic compounds that will have "therapeutic" benefits and their performance efficacy assessment are essentially missing in the field of aromatherapeutic textiles. Many research studies mentioned the term "aromatherapeutic textiles", even though the authors of such papers never measured the "therapeutic" benefit of such aroma-textiles. These studies did not include clinical trials with a suitable experimental protocol that measures the intended therapeutic effects of essential oils such as decreased anxiety, pain, heart rate, or improved sleep, for instance. 


\subsection{Techniques and Examples of Microencapsulated EO on Textiles}

There are several techniques used by researchers to embed EO microcapsules on textile materials, such as screen printing [23,24], the impregnation method [23], pad-drycure $[20,22,25,26]$ and polymeric nanoparticle formation [27-29]. Each technique has its own advantages and effects on the mechanical properties of the material being finished. Melamine-formaldehyde (MF) polymer wall capsules were used to encapsulate lavender, rosemary, and sage essential oils in a study that examined fragrance finishing of cotton [23]. In a surface morphology comparison of the treated textiles, both impregnation and screenprinting techniques produced comparable results and both were deemed appropriate for fragrant clothing [23]. While screen printing was found to provide controlled application on specific target areas of the fabric, which is an advantage, it lowered the air permeability and flexure of the fabric. A further study [24] utilizing the screen printing of (MF) microcapsules to examine separate cores of (1) a mixture of lavender, rosemary, and sage essential oils; (2) the antimicrobial triclosan (TCS) and (3) the fire-retardant try phenyl phosphate (TPP) was undertaken to achieve a fabric with lasting aroma, antimicrobial and flame-retardant properties. The printing of the MCs impacted the mechanical properties of the fabrics but there were no differences in mechanical properties between aroma, antimicrobial, or fire-retardant MCs. All treated fabric samples showed very good durability during washing. This study demonstrated that printing of melamine formaldehyde (MF) MCs can improve the functionality of textiles and may represent a universal approach to explore going forward.

Further research was conducted toward the use of MF microencapsulated essential oils in the cosmetics industry [20]. Rose and sage microcapsules were deposited by padding on $100 \%$ cotton and $50 \%$ cotton $/ 50 \%$ polyester woven fabrics. An acrylate-based binder was used to fix the microcapsules to the fabric. The incorporation of a binder in this process can improve the fabric's durability through washing and handling [24]. While physical and mechanical tests were performed and revealed minimal influence of the treatments on the fabrics, the major contribution of this study was the evaluation of biological properties. Human skin cells were used for in vitro biocompatibility assessment of the rose- and sage-microencapsulated fabric and confirmed the absence of cytotoxicity after short-term exposure. These findings suggest that fabrics with microencapsulated rose and sage immobilized on them may be good candidates for providing biological properties such as antioxidant, anti-inflammatory, and antibacterial effects for the wearer [20]. Further research in this area is warranted to examine these effects.

Melamine-formaldehyde (MF) and gelatine-carboxymethylcellulose (CMC) as wall materials were used for comparison in a study examining microencapsulated limonene intended as a fragrance for footwear [28]. The comparison demonstrated that the MF microcapsules were more resistant to the thermal and mechanical stresses that footwear would undergo during manufacturing, while the $\mathrm{CMC}$ microcapsules were more sensitive and may rupture under those conditions. It was suggested that the CMC microcapsules might still be incorporated into shoe components such as insoles after the more rigorous shoemaking process is completed.

\subsection{Environmental Considerations}

Nanoparticles (NP) are colloid-sized particles with diameters ranging from 10 to $1000 \mathrm{~nm}$, whereas microcapsules have diameters between a few micrometers and a few millimeters $[29,30]$. Nanoscale materials are advantageous in microcapsules because they have a higher specific surface that enables more efficient fragrance loading in the cavity of the carrier while optimizing the interaction with the fragrance and its sustained release [27]. However, concerns regarding the accumulation of microplastics in the environment and pending regulations by the European Chemicals Agency (ECHA) have challenged researchers to design polymeric NPs that can demonstrate high adsorption to the textile and low or no desorption when it undergoes scrubbing and recurring washing. With this challenge in mind, researchers synthesized polymeric NPs with different 
physicochemical properties including surface charge, glass transition temperature, size and degree of cross-linking to examine how they affect the NP adsorption on a $90 \%$ Cotton and $10 \%$ elastane textile [27]. Surface charge was found to be the most important parameter in adsorption, while the degree of cross-linking had little to no effect. Transition temperature demonstrated that soft PBA NPs adsorbed more than the harder PBMA and PSTY NPs. Regarding size, bigger NPs were better for adsorption but worse in terms of encapsulation efficiency. Trials such as these demonstrate the importance of developing nanoparticles that maximize the interaction between the carrier and the EO encapsulated in the core to avoid NP desorption from the textile and further contamination of the environment with nanoplastics.

There is a continuing effort to develop a system for fragrant textiles using nontoxic and planet-friendly shell materials to encapsulate fragrances that will last through consumer wear and care. The use of bio-based capsules to contain essential oils is a strategy in this direction. Gelatin-arabic gum (GAM) and yeast cell microcapsules (YCM) are lowcost, readily available, safe for human use and non-toxic to the environment, making them suitable containers for essential oils. Researchers found GAM microcapsules to be advantageous over YCM for initial fragrance intensity, fragrance-controlled release and washing durability [25]. GAM was also found to be effective in the controlled release of citronella oil from wool [26]. In another study, plant-based alginate nanocapsules of peppermint oil were used as an antimicrobial for cotton fabrics and found to be effective for reducing E. coli and $S$. aureus bacteria while also being durable over repeated washing while maintaining their aroma [29].

Not all synthetic polymers are toxic to the environment. For example, methyl methacrylate styrene copolymer served as shell material for a cologne essential oil nanocapsule created by miniemulsion polymerization [28]. The cologne was created from a combination of lemon oil, rosemary oil and bergamot oil. In this study, the prepared nanocapsules had good thermostability and washing durability, demonstrating positive potential as a treatment for enhancing fragrance durability in functional textiles.

\subsection{Use of Cyclodextrin and Aromatic Componds}

Another encouraging and more recent contender for a molecular container with the capability for sustained release of an EO from a textile substrate that also overcomes some of the environmental and health-related issues are cyclodextrins [22,31-33]. They provide a unique physical structure that has a hydrophilic exterior and a hydrophobic interior cavity, which makes them highly suitable to form inclusion complexes with EOs. They are especially useful in applications in the food, pharmaceutical and agricultural industries due to their biocompatibility and non-toxic nature. The fact that cyclodextrins are also regulated by the US Food and Drug Administration makes them a highly desirable compound for research purposes. Fabrics finished with $\beta$-Cyclodextrin and essential oils using the sol-gel method were evaluated by human panels in two studies [22,34]. Lavender and Cedarwood were found to be an effective remedy for stress in the first study that had subjects smell fabric specimens containing the essential oils as compared to a control fabric during an experimental protocol that involved inducing stress on the subjects [22]. In the second study, the intensity of eucalyptus, lavender and lemon fragrances was found to decrease after launderings, but a higher scent intensity was found on a poly/cotton fabric as compared to a cotton fabric. The result was attributed to a higher add-on of the inclusion compound to the poly/cotton [34].

While the use of $\beta$-Cyclodextrin is advantageous in prolonging the release of essential oils, a modified form of it, monochlorotriazine $-\beta$-Cyclodextrin (MCT- $\beta C D)$, has been shown to be a more favorable host for functionalizing fabrics [35]. Eucalyptus, peppermint, lavender, jasmine, clove and cedarwood anchored with MCT- $\beta$ CD on cotton were shown to have enhanced oil retention in washed fabrics over those anchored with $\beta C D$ alone. Furthermore, no major changes were found in tensile strength, stiffness or air permeability. 


\subsection{Challenges and Opportunities in the Delivery of Aromatic Compounds}

Challenges remain in the delivery of aromatic compounds in textiles and further research is needed on how the physiochemical properties of the various natural and synthetic polymers impact the adsorption of nanoparticles on textiles. The laundering durability of such finishes needs to be investigated. Further investigating the combination of EOs and active compounds to increase their potency, prolonged release and other potential functionalities will serve to stimulate applications for a variety of therapeutic functions. It is expected that continued multidisciplinary applications of microencapsulation and nanoencapsulation of essential oils will help to overcome many of these challenges and enhance future opportunities to improve human health and well-being. Finally, with cyclodextrins offering the advantages of eco-friendliness, controlled release of substance, and being an FDA regulated compound, their continued use and experimentation with aromatic compounds will encourage a host of future textile developments.

\section{Medical Textiles}

The scope and usefulness of bio-engineered products have increased tremendously in the recent past and textile materials offer an excellent medium to carry these bio-engineered products for human health and welfare [47]. Their niche application specifically in the areas of the medical and defense industries have caught the attention of both researchers and industry. Medical textiles have two broad approaches as (I) Implantable devices, and (II) Bio-functional textiles. The former includes vascular prostheses, suture threads, stents, substitutes of tendons, etc., and the latter includes textiles with value-added function [36].

\subsection{Bio-Functional Textiles}

The bio-functional textiles have several functions including but not limited to antibacterial, antiviral, anti-inflammatory, and biomedical applications such as wound dressing, healing, and organ-regenerative properties [48]. They can also be used for filtering, protection, organ strengthening, replacing failing organs, supporting tissue regeneration and preventing post-operative complications, applications which have been useful in the pharmacological industry. Due to the prolonged and close contact of fabric with the skin, textile materials can be an excellent drug delivery system.

Apart from their usual function of providing a protective layer to the body, these bio-functional textiles protect the wearer from environmental pathogens and can have a direct therapeutic effect to the body. For example, a pharmacological drug or plant-based active ingredient can be delivered from textiles directly to the body and these therapeutic textiles can provide an effective therapeutic function to the wearer, such as "antiallergic" or "antipsoriasis" effects. The essential oil-based bio-functional textiles can be used either for biocidal (antibacterial, antiviral, antifungal and insect repellent) applications or therapeutic effects $[37,48]$. Rose and sage essential oils present the highest applicability in the functions of biocidal and traditional medicine $[39,40]$. The dominant compound of sage essential oil, 1,8-cineole, is known for its antifungal and antimicrobial properties, while rose EO has shown efficacy against anxiety, depression, and stress-related conditions [41].

In another study, bio-active textiles were impregnated with econazole nitrate (ECN), an active ingredient for the treatment of fungal infections [42]. The thermosensitive microcapsules loaded with ECN released the drug during contact with the skin. The antifungal activity was reported to be comparable to the topical application of the ECN-based topical cream for fungal infection treatment. The ECN-based textiles were reported to show antifungal activity against a wide-ranging fungal strain in vitro and in vivo. These textiles enable high therapeutic efficacy against Cutaneous candidiasis in mice. Such pharmacological applications of textiles show a promising future for biomedical textiles for treating fungus-related skin conditions. 
Textiles coated with esterificated citric acid also brought about significant improvement in atopic eczema symptoms in both objective and subjective analyses of 20 patients [43]. Over the last decade, there have been several new developments in the therapeutic potential of textiles such as silver-coated textiles, seaweed-infused fabrics and chitosan-impregnated textiles as an alternative to medical treatment in milder cases of skin conditions such as atopic eczema and other fungal infections.

\subsection{Biocidal Applications}

The EO obtained from Iryanthera polyneura (an Amazonian plant commonly known as cumala-colorada) were studied for their antimicrobial activity against selected pathogens [44] such as Candida albicans, Enterococcus faecalis, Escherichia coli, Pseudomonas aeruginosa, Staphylococcus aureus, Streptococcus mutans and S. sanguinis. Spathulenol, $\alpha$-cadinol and $\tau$-muurolol were major components of the EO. The oil showed promising antimicrobial activity against E. faecalis, S. aureus and PC-3, when collected in the dry season. However, the oils obtained from leaves collected in the rainy season were more active against $S$. mutans and $S$. sanguinis. The antibacterial activities of the essential oils from the leaves of I. polyneura are related to seasonal climate variation and are influenced by compounds that are minor components of the oils. Another example of such bio-functional textiles is mosquito repellent textile. Mosquitoes such as Anopheles Meigen, Culex L. and Haemagogus L. are responsible for the transmission of several tropical diseases such as malaria, hemorrhagic dengue, yellow fever and filariasis. Traditional sources of mosquito repellents can be found in plant-based essential oils, and there are currently 144 active patents in this area [38]. Essential oils reported to assist the mosquito repellent function include cinnamon, citronella, eucalyptus, lavender, peppermint, clove, lemongrass, germanium, camphor, lemon, chamomile, jasmine, juniper, verbena and wild soybean. Textiles infused with essential oils to perform a mosquito repellent function are not only effective in their intended function, but also provide a sustainable approach to repel mosquitoes.

\subsection{Therapeutic Applications}

Value-added functional textiles for human well-being and comfort continue to increase in popularity, particularly the aromatherapy-based textile treatments that manipulate our olfactory senses and provide therapeutic benefits. The essential oils are generally microencapsulated and coated on textile surfaces to develop functional textiles with medicinal and antimicrobial properties [45]. For instance, lemon oil infused textiles provided psychological and sensorial comfort, while patchouli oil, Artemsia argyi oil and moxa oil, when applied to textiles, showed ecofriendly antibacterial activity [46]. It is believed that such antibacterial textiles provide a therapeutic function to heal infection-induced skin diseases and create a wound-healing mechanism to improve the overall health of the skin. However, biocidal effects, such as antibacterial/antiviral/antifungal effects, are more popular applications when it comes to biofunctional textiles infused with micro/nanoecapsulated EOs. In this literature review, we found that there is rarely an emphasis on the "therapeutic" effect of the aromatherapeutic textiles. Further research using clinical trials with a focus on assessing the therapeutic benefits of aromatherapeutic textiles is needed.

\section{Conclusions}

This review of the literature on aromatherapy and textiles revealed a host of studies from a wide variety of fields that can collectively be seen as encouraging to the premise that textiles may be a suitable medium for enhancing the human experience when worn and embedded with essential oils. Historically, textiles have been referred to as a second skin [49], a portable environment [50] and as "omnipresent, pervasive and ... interwoven into the private and public practices of every culture, both formal and informal ... " [51].

In the 21st century, textiles as our nearest environment have demonstrated the potential to serve as a conduit for the transdermal delivery and controlled release of a host of health-related compounds to patients and individuals for enhancing health and well-being. 
Innovations in imprinting or embedding textiles with bioactive compounds are leading to dermal treatments for bacterial, allergic, fungal, and other compromising health conditions. Some of the suggested applications for such textiles are antibacterial masks, bacteriostatic sheets, and healthcare apparel. The potential for health-related textile products is enormous but more research is needed to take the current state of knowledge forward to define the specific therapeutic benefits of the EOs utilized.

The gaps that remain in the broad and diverse disciplines conducting research on aromatherapy and textiles will need to narrow in order for the growth of this industry and the development of products that will bring comfort and well-being to the population to continue. Two of the major gaps that exist at the present time are highlighted below:

1. Studies in the area of aromatherapy are generally using the traditional application of essential oils via massage therapy to understand the therapeutic benefit of EOs. Upon topical application, EOs release their aroma with a burst, which quickly dissipates. There is no focus on optimizing the dosage, control of the EO release rate or the delivery mechanism. These studies are vexed with several issues and the results are rarely reproducible, which poses questions on their scientific worth.

2. On the other hand, studies which focused on achieving the controlled release, EO concentration optimization, and delivery mechanism such as micro/nanoencapsulation for controlled release of aroma are focused truly on the encapsulation mechanism and not on assessing the "therapeutic" benefit of such aromatherapeutic textiles.

It has been suggested that the textile industry needs to evolve due to consumer expectations and intense product competition [34]. Further research on the fusion of EOs, aromatherapy, and textiles is posed to inspire such an evolution. However, in order to overcome the gaps in knowledge at this point, synergy between related fields of knowledge must come about to better understand the efficacy of aromatherapeutic textiles.

Funding: This research was funded by the National Institute of Food and Agriculture, U.S. Department of Agriculture, Hatch project under WVA00742.

Institutional Review Board Statement: Not applicable.

Informed Consent Statement: Not applicable.

Data Availability Statement: Not applicable.

Conflicts of Interest: The authors declare no conflict of interest. The funders had no role in the design of the study; in the collection, analyses, or interpretation of data; in the writing of the manuscript, or in the decision to publish the results.

\section{References}

1. Ghayempour, S.; Montazer, M. Micro/Nanoencapsulation of Essential Oils and Fragrances: Focus on Perfumed, Antimicrobial, Mosquito-Repellent and Medical Textiles. J. Microencapsul. 2016, 33, 497-510. [CrossRef] [PubMed]

2. Tisserand, R.B. The Art of Aromatherapy, the Healing and Beautifying Properties of the Essential Oils of Flowers and Herbs; Healing Arts Press: Fairfield, CT, USA, 1977.

3. Damian, P.; Damian, K. Aromatherapy: Scent and Psyche: Using Essential Oils for Physical and Emotional Well-Being; Healing Arts Press: Fairfield, CT, USA, 1995.

4. Lawless, M.K.; Mathies, R.A. Excited-state Structure and Electronic Dephasing Time of Nile Blue from Absolute Resonance Raman Intensities. J. Chem. Phys. 1992, 96, 8037-8045. [CrossRef]

5. Gattefosse, R.M. Gattefosse's Aromatherapy; Tisserand, R.B., Ed.; C.W. Daniel Company Limited: Saffron Walden, UK, 1996.

6. Cooksley, V.G. Aromatherapy: Soothing Remedies to Restore, Rejuvenate and Heal; Prentice Hall: Hoboken, NJ, USA, 2002.

7. Farrar, A.J.; Farrar, F.C. Clinical Aromatherapy. Nurs. Clin. N. Am. 2020, 55, 489-504. [CrossRef] [PubMed]

8. Hwang, E.; Shin, S. The Effects of Aromatherapy on Sleep Improvement: A Systematic Literature Review and Meta-Analysis. J. Altern. Complement. Med. 2015, 21,61-68. [CrossRef]

9. Pace, S. Essential Oils in Hospitals: The Ethics, Safety, Cost and Application of Clinical Aromatherapy. Available online: https://www.tisserandinstitute.org/essential-oils-in-hospitals/ (accessed on 14 October 2021).

10. Swamy, M.K.; Akhtar, M.S.; Sinniah, U.R. Antimicrobial Properties of Plant Essential Oils against Human Pathogens and Their Mode of Action: An Updated Review. Evid.-Based Complement. Altern. Med. 2016, 2016, 3012462. [CrossRef] 
11. Forrester, L.T.; Maayan, N.; Orrell, M.; Spector, A.E.; Buchan, L.D.; Soares-Weiser, K. Aromatherapy for Dementia. Cochrane Database Syst. Rev. 2014, 2, 1-62. [CrossRef] [PubMed]

12. Agatonovic-Kustrin, S.; Chan, C.K.Y.; Gegechkori, V.; Morton, D.W. Models for Skin and Brain Penetration of Major Components from Essential Oils Used in Aromatherapy for Dementia Patients. J. Biomol. Struct. Dyn. 2020, 38, 2402-2411. [CrossRef]

13. Lakhan, S.E.; Sheafer, H.; Tepper, D. The Effectiveness of Aromatherapy in Reducing Pain: A Systematic Review and MetaAnalysis. Pain Res. Treat. 2016, 2016, 8158693. [CrossRef]

14. Song, J.-A.; Lee, M.; Min, E.; Kim, M.-E.; Fike, G.; Hur, M.-H. Effects of Aromatherapy on Dysmenorrhea: A Systematic Review and Meta-Analysis. Int. J. Nurs. Stud. 2018, 84, 1-11. [CrossRef]

15. Lee, M.S.; Choi, J.; Posadzki, P.; Ernst, E. Aromatherapy for Health Care: An Overview of Systematic Reviews. Maturitas 2012, 71, 257-260. [CrossRef]

16. Won, S.-J.; Chae, Y.-R. The Effects of Aromatherapy Massage on Pain, Sleep, and Stride Length in the Elderly with Knee Osteoarthritis. J. Korean Biol. Nurs. Sci. 2011, 13, 142-148.

17. Tanvisut, R.; Traisrisilp, K.; Tongsong, T. Efficacy of Aromatherapy for Reducing Pain during Labor: A Randomized Controlled Trial. Arch. Gynecol. Obstet. 2018, 297, 1145-1150. [CrossRef] [PubMed]

18. Son, H.K.; So, W.-Y.; Kim, M. Effects of Aromatherapy Combined with Music Therapy on Anxiety, Stress, and Fundamental Nursing Skills in Nursing Students: A Randomized Controlled Trial. Int. J. Environ. Res. Public Health 2019, 16, 4185. [CrossRef]

19. Desborough, M.J.R.; Keeling, D.M. The Aspirin Story-From Willow to Wonder Drug. Br. J. Haematol. 2017, 177, 674-683. [CrossRef]

20. Stan, M.S.; Chirila, L.; Popescu, A.; Radulescu, D.M.; Radulescu, D.E.; Dinischiotu, A. Essential Oil Microcapsules Immobilized on Textiles and Certain Induced Effects. Materials 2019, 12, 2029. [CrossRef]

21. West, A.J.; Annet-Hitchcock, K.E. A Critical Review of Aroma Therapeutic Applications for Textiles. J. Text. Appar. Technol. Manag. 2014, 9, 1-13.

22. Mehta, S.; MacGillivray, M.S. Analysis of Stress Relief Effect by Use of Aroma-Therapeutic Textiles. AATCC J. Res. 2016, 3, 38-44. [CrossRef]

23. Golja, B.; Sumiga, B.; Tavcer, P.F. Fragrant finishing of cotton with microcapsules: Comparison between printing and impregnation. Color. Technol. 2013, 129, 338-346. [CrossRef]

24. Golja, B.; Tavčer, P.F. Textile Functionalization by Printing Fragrant, Antimicrobial and Flame-Retardant Microcapsules. Tekstilec 2016, 59, 278-288. [CrossRef]

25. Specos, M.M.M.; Escobar, G.; Marino, P.; Puggia, C.; Defain Tesoriero, M.V.; Hermida, L. Aroma Finishing of Cotton Fabrics by Means of Microencapsulation Techniques. J. Ind. Text. 2011, 40, 13-32. [CrossRef]

26. Bezerra, F.M.; Carmona, O.G.; Carmona, C.G.; Lis, M.J.; De Moraes, F.F. Controlled Rrelease of the Essential Oil of Citronella Microencapsulated using Cotton and Polyester Matrices. Cellulose 2016, 23, 1459-1470. [CrossRef]

27. Manfredini, N.; Ilare, J.; Invernizzi, M.; Polvara, E.; Contreras Mejia, D.; Sironi, S.; Moscatelli, D.; Sponchioni, M. Polymer Nanoparticles for the Release of Fragrances: How the Physicochemical Properties Influence the Adsorption on Textile and the Delivery of Limonene. Ind. Eng. Chem. Res. 2020, 59, 12766-12773. [CrossRef]

28. Liu, C.; Liang, B.; Shi, G.; Li, Z.; Zheng, X.; Huang, Y.; Lin, L. Preparation and Characteristics of Nanocapsules Containing Essential Oil for Textile Application. Flavour Fragr. J. 2014, 30, 295-301. [CrossRef]

29. Ghayempour, S.; Mortazavi, S.M. Microwave Curing for Applying Polymeric Nanocapsles Containing Essential Oils on Cotton Fabric to Produce Antimicrobial and Fragrant Textiles. Cellulose 2015, 22, 4065-4075. [CrossRef]

30. Sánchez-Navarro, M.M.; Pérez-Limiñana, M.Á.; Arán-Ais, F.; Orgilés-Barceló, C. Scent Properties by Natural Fragrance Microencapsulation for Footwear Applications. Polym. Int. 2015, 64, 1458-1464. [CrossRef]

31. Suganya, V.; Anuradha, V. Microencapsulation and Nanoencapsulation: A Review. Int. J. Pharm. Clin. Res. 2017, 9, 233-239. [CrossRef]

32. Shrimali, E.; Dedhia, E.M. Microencapsulation for Textile Finishing. IOSR J. Polym. Text. Eng. 2015, 2, 1-4. Available online: https:/ / www.iosrjournals.org/iosr-jpte/papers/Vol2-issue2/A0220104.pdf (accessed on 14 June 2021).

33. Khajavi, R.; Ahrari, M.; Toliyat, T.; Bahadori, L. Molecular Encapsulation of Lavender Essential Oil by B-Cyclodextrin and Dimethyl Dihydroxy Ethylene Urea for Fragrance Finishing of Cotton Fabrics. Asian J. Chem. 2013, 25, 459-465. [CrossRef]

34. Ghosh, S.; Chipot, N. Embedding Aromatherapy Essential Oils into Textile Fabric Using $\beta$-Cyclodextrin Inclusion Compound. Indian J. Fibre Text. Res. 2015, 40, 140-143.

35. Khanna, S.; Sharma, S.; Chakraborty, J.N. Performance Assessment of Fragrance Finished Cotton with Cyclodextrin Assisted Anchoring Hosts. Fash. Text. 2015, 2, 19. [CrossRef]

36. Radu, C.-D.; Parteni, O.; Ochiuz, L. Applications of Cyclodextrins in Medical Textiles-Review. J. Control. Release 2016, 224, 146-157. [CrossRef]

37. McLaren, R.; Joseph, F.; Baguley, C.; Taylor, D. A Review of e-Textiles in Neurological Rehabilitation: How Close Are We? J. Neuroeng. Rehabil. 2016, 13, 59. [CrossRef]

38. Pohlit, A.M.; Lopes, N.P.; Gama, R.A.; Tadei, W.P.; de Andrade Neto, V.F. Patent literature on mosquito repellent inventions which contain plant essential oils-A review. Planta Med. 2011, 77, 598-617. [CrossRef]

39. El Asbahani, A.; Miladi, K.; Badri, W.; Sala, M.; Aït Addi, E.H.; Casabianca, H.; Mousadik, A.E.; Hartmann, D.; Jilale, A.; Renaud, F.N.R.; et al. Essential Oils: From Extraction to Encapsulation. Int. J. Pharm. 2015, 483, 220-243. [CrossRef]

40. Chrysargyris, A.; Panayiotou, C.; Tzortzakis, N. Nitrogen and Phosphorus Levels Affected Plant Growth, Essential Oil Composition and Antioxidant Status of Lavender Plant (Lavandula angustifolia Mill.). Ind. Crops Prod. 2016, 83, 577-586. [CrossRef] 
41. Ceylan, R.; Zengin, G.; Uysal, S.; Ilhan, V.; Aktumsek, A.; Kandemir, A.; Anwar, F. GC-MS Analysis and in Vitro Antioxidant and Enzyme Inhibitory Activities of Essential Oil from Aerial Parts of Endemic Thymus spathulifolius Hausskn. et Velen. J. Enzym. Inhib. Med. Chem. 2016, 31, 983-990. [CrossRef] [PubMed]

42. Hossain, M.A.; Lalloz, A.; Benhaddou, A.; Pagniez, F.; Raymond, M.; Le Pape, P.; Simard, P.; Théberge, K.; Leblond, J. Econazole Imprinted Textiles with Antifungal Activity. Eur. J. Pharm. Biopharm. 2016, 101, 137-144. [CrossRef]

43. Jaeger, T.; Rothmaier, M.; Zander, H.; Ring, J.; Gutermuth, J.; Anliker, M. Acid-Coated Textiles (PH 5.5-6.5)—A New Therapeutic Strategy for Atopic Eczema? Acta Derm.-Venereol. 2015, 95, 659-663. [CrossRef] [PubMed]

44. Martins, E.R.; Díaz, I.E.; Paciencia, M.L.; Fana, S.A.; Morais, D.; Eberlin, M.N.; Silva, J.S.; Silveira, E.R.; Barros, M.P.; Suffredini, I.B. Interference of Seasonal Variation on the Antimicrobial and Cytotoxic Activities of the Essential Oils from the Leaves of Iryanthera polyneura in the Amazon Rain Forest. Chem. Biodivers. 2019, 16, e1900374. [CrossRef] [PubMed]

45. Lam, P.L.; Li, L.; Yuen, C.W.M.; Gambari, R.; Wong, R.S.M.; Chui, C.H.; Lam, K.H. Effects of multiple washing on cotton fabrics containing berberine microcapsules with anti-Staphylococcus aureus activity. J. Microencapsul. 2013, 30, 143-150. [CrossRef]

46. Hu, L.; Du, M.; Zhang, J.; Wang, Y. Chemistry of the Main Component of Essential Oil of Litsea cubeba and its Derivatives. Open J. For. 2014, 4, 457-466.

47. Bernard, H.R. Research Methods in Anthropology: Qualitative and Quantitative Approaches; Rowman \& Littlefield: Lanham, MD, USA, 2017.

48. Martel, B.; Campagne, C.; Behary Massika, N. Quand Les Textiles Vous Soignent. Med. Sci. 2017, 33, 73-80. [CrossRef] [PubMed]

49. Flint, I. Second Skin: Choosing and Caring for Textiles and Clothing; Murdoch Books: Millers Point, Australia, 2012.

50. Watkins, S.; Dunne, L. Functional Clothing: From Sportswear to Spacesuits; Bloomsbury: London, UK, 2015.

51. Kokoli, A. Creative Tensions: Making (it), Unmaking, and Making do in Textiles informed by Feminism. In A Companion to Textile Culture; Harris, J., Ed.; Wiley-Blackwell: Hoboken, NJ, USA, 2020; p. 226. 